Yu. T. Tsap, A. V.Stepanov, Yu. G. Kopylova

"On the Description of Transverse Wave Propagation Along Thin Magnetic Flux Tubes"

Geomagnetism and Aeronomy, 2018, V. 58, N 7, pp.942-946.

DOI: $10.1134 / \mathrm{S} 001679321807023 \mathrm{X}$

\title{
ON THE DESCRIPTION OF TRANSVERSE WAVE PROPAGATION ALONG THIN MAGNETIC FLUX TUBES
}

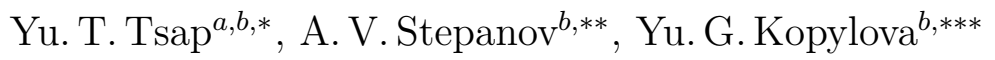 \\ ${ }^{a}$ Crimean Astrophysical Observatory of the Russian Academy of Sciences, Crimea, Russia \\ ${ }^{b}$ Pulkovo Observatory of the Russian Academy of Sciences, St. Petersburg, Russia \\ *email:yur_crao@mail.ru, ${ }^{* *}$ email: stepanov@gaoran.ru, ${ }^{* * *}$ email: yul@gaoran.ru
}

Two approaches are used for description of linear transverse (kink) modes excited in a vertical thin magnetic flux tube. First one is based on the elastic thread model (Spruit, 1981). The second one follows from the the Taylor and Laurent series expansions of wave variables with respect to the tube radius inside and outside of the magnetic flux tube (Lopin and Nagorny, 2013). It has been shown that the main reason of the discrepancy of these approaches is related to the phenomenological equation of plasma motion used in the former case. This suggests that results obtained on the basis of this equation should be revised.

\section{Introduction}

It is well known that the magnetic flux tubes formed under action of convective motions can play an important role in the solar atmosphere. According to existing concepts the tubes with diameters of about $100 \mathrm{~km}$ or less are the main structural elements of the solar photosphere (Stenflo, 2011; Ji et al., 2012; Sharvkin and Kosovichev, 2014) and they can contain up to 90 per cent of the total magnetic field (Howard and Stenflo, 1972; Rüedi et al., 1992). These magnetic structures transmit the convective energy of plasma motion from the photosphere to the corona like waveguides. The last property is the subject of investigation presented in this article.

It is well established now that the high frequency Alfvén type modes (kink, torsional) generated in the photosphere are strongly damped in the chromosphere (De Pontieu et al., 2001; Leake et al., 2005; Soler et al., 2017) if their periods $T<10 \mathrm{~s}$ while the waves with $T>40 \mathrm{~s}$ are quite strongly reflected in the transition region (Tsap, 2006; Soler et al., 2017). This suggests that waves with $T=10-40$ s can be responsible for heating at least of lower corona (see also Gelfreikh et al., 2004). In turn, there are many indications that linear kink waves with $T>1$ min excited in magnetic flux tubes of the solar photosphere (Fujimura and Tsuneta, 2009; Jess et al., 2017) are a prime candidate for heating and accelerating the fast wind because they have the ability to transport energy over large distances in the corona (Morton et al., 2015). However, the possibility of effective generation and propagation of these waves in the photosphere is still unclear.

The theory of linear kink modes based on the thin magnetic flux tube approximation for the first time was proposed independently by Ryutov and Ryutova (1976) and Spruit (1981). Since the approach of Ryutov and Ryutova (1976) suggests the constant cross section of the tube we will not discuss it further in detail. The approach proposed by Spruit (1981) is more general and it is often used up to now to description of magnetic flux tube dynamics in the solar stellar convection 
zone (Fan, 2009; Weber and Browning, 2016). Note that the thin flux tube approximation can also describe MHD waves in the solar photosphere and lower chromosphere (Lopin and Nagorny, 2017) quite well.

One of the main problem of this approach connected with the phenomenological equation of motion. Fist of all, this concerns the effect of the enhanced inertia caused by the backreaction of the the ambient medium to the relative motion of the flux tube (Spruit, 1981). The correct treatment of this contribution is a source of continuing controversy (Choudhuri, 1990; Cheng, 1992; Fan. Fisher. and McClvmont, 1994; Moreno-Insertis, Ferriz-Mas, and Schlüssler, 1996; Longcope and Klapper 1997; Osin. Volin, and Ulmschneider, 1999).

In order to describe the MHD waves in the thin magnetic flux tubes approximation in addition to the Spruit's approach the second method based on the power series expansion of wave variables with respect to the tube radius (see also Roberts and Webb, 1978; Ferriz-Mas and Schüssler, 1989) is used. Recently, Lopin et al. (2014), based on the system of linearized ideal MHD equations and using this method have shown that the phenomenological equation of motion can be used for the description of kink modes in thin magnetic flux tubes if we take into account the radial component of the magnetic flux tube in the induction equation. This means that the phenomenological equation of motion can be used for the description of MHD waves excited in thin magnetic flux tubes when the relative motion of the ambient medium is equal to zero.

This study is devoted to the analysis of the phenomenological equation of motion proposed by proposed by Spruit (1981) based on the the power series expansion of wave variables for linear kink mode propagation in a vertically stratified thin magnetic flux tube.

\section{Vertical non-twisted magnetic flux tubes in stratified atmosphere and their properties}

Let us first slightly generalize results obtained earlier for a vertical isolated magnetic flux tube in the stratified atmosphere with the radius $a$ (Roberts and Webb, 1978; Hollweg, 1981; Ferriz-Mas and Schüssler, 1989).

The equilibrium magnetic field of an axisymmetric untwisted flux tube in cylindrical coordinates can be written as

$$
\mathbf{B}=B_{z}(z) \mathbf{e}_{z}+B_{r}(r, z) \mathbf{e}_{r},
$$

where $\mathbf{e}_{z}$ and $\mathbf{e}_{r}$ are the unit vectors.

For the sake of simplicity we can assume that the radial component of its magnetic field due to the tube expansion with height is

$$
B_{r}(r, z)=\alpha r, \quad \alpha=\text { const. }
$$

This suggestion can be considered as some generalization of the thin flux tube approximation since $\alpha$ can take arbitrary values. Note that in the thin magnetic flux tube approximation $B_{z}(r, z)$ and $B_{r}(r, z)$ components of the tube magnetic field are expanded in a Taylor series (e.g., Ferriz-Mas and Schüssler, 1989; Lopin and Nagorny, 2013)

$$
\begin{gathered}
B_{z}(r, z)=B_{z}(0, z)+r^{2} \frac{\partial B_{r}^{2}}{\partial r^{2}}(0, z), \\
B_{r}(r, z)=B_{r}(0, z)+r \frac{\partial B_{r}}{\partial r}(0, z),
\end{gathered}
$$


where $B_{z}(0, z) \equiv B_{z}(z)$ is independent of $r$ and $B_{r}(0, z)=0$ in the lowest order of the series expansion, and $\left|B_{r}(a, z)\right| /\left|B_{z}(z)\right| \ll 1$.

The equation of the magnetic field lines, in view of equation (2), can be reduced to the relationships

$$
\frac{d z}{d r}=\frac{B_{z}}{B_{r}}=\frac{B_{z}}{\alpha r}
$$

Since the magnetic field lines are closed curves $(\nabla \cdot \mathbf{B}=0)$, using equation (2), we get

$$
\nabla \cdot \mathbf{B}=\frac{1}{r} \frac{\partial\left(r B_{r}\right)}{\partial r}+\frac{\partial B_{z}}{\partial z}=2 \alpha+\frac{\partial B_{z}}{\partial z}=0
$$

Hence, equations (11), (2)), and (ㅁ) (Roberts and Webb, 1978; Ferriz-Mas and Schüssler, 1989) give

$$
B_{r}(r, z)=-\frac{r}{2} \frac{d B_{z}(z)}{d z}
$$

Combining equations (5) and (6), we obtain the differential equation in the form

$$
2 \frac{d r}{r}+\frac{d B_{z}}{B_{z}}=0
$$

The general solution of equation (8) can be represented as

$$
B_{z} r^{2}=\text { const. }
$$

Equation (9) means that the longitudinal magnetic flux $F_{z}=B_{z} a^{2}$ of the thin or wide tube is conserved. In particularly, this important circumstance was used by Hollweg (1981) (see also Hollweg, 1984) and Routh et al. (2007) for description of torsional modes in a wide magnetic flux tube. However, in the future we shall confine ourselves to the study of thin flux tubes.

The plasma density in stratified atmosphere decreases with the hight $z$ inside and outside $(e)$ the tube for the isothermal atmosphere according to the barometric formulae

$$
\rho=\rho_{0} e^{-z / H}, \quad \rho_{e}=\rho_{0 e} e^{-z / H},
$$

where $H$ is a characteristic scale hight. In turn, the balance of total pressures, which follows from the integration of the MHD equilibrium equation, for an isolated $\left(B_{e}=0\right)$ magnetic flux tube is

$$
p+\frac{B^{2}}{8 \pi}=p_{e}
$$

Equations (10) and (11) give

$$
B_{z} \propto e^{-z / 2 H}, \quad B_{r} \propto e^{-z / 2 H} .
$$

Hence, equations (7) and (12) implies that the radial component of a magnetic flux tube is

$$
B_{r}(r, z)=\left(\frac{r}{4 H}\right) B_{z}(r, 0) e^{-z / 2 H} .
$$

Equations (9), (12), and (13) describe the dependence of the magnetic field on $r$ and $z$ in thin vertical magnetic flux tubes. Besides, equation (13) suggests that the thin magnetic flux tube approximation $\left(B_{r} \ll B_{z}\right)$ is fulfilled if the tube radius $(a \ll 4 H)$. For example, taking in the photosphere $H=150 \mathrm{~km}$, we find $a \ll 600 \mathrm{~km}$ (see also Lopin and Nagorny, 2017). 


\section{The phenomenological equation of motion and the elastic thread model proposed by Spruit (1981)}

The approach proposed by Spruit (1981) is based the linearized phenomenological equation of motion, which using standard notation can be represented as

$$
\left(\rho+\rho_{e}\right) d \mathbf{v}_{\perp} / d t=\delta \mathbf{F}_{\perp}
$$

Equation (14) seems to be the main disadvantage of Spruit's approach. Indeed, the transverse force $\delta \mathbf{F}_{\perp}$ on the right-hand side of equation (14) includes the action of the internal force only while the left-hand side corresponds to the plasma inertia inside and outside $(e)$ the magnetic flux tube.

Spruit (1981) took formally into consideration external forces using the the pressure balance equation for disturbed quantities

$$
\delta P=\delta p_{e},
$$

where the total pressure $P=p+B^{2} /(8 \pi)$. Indeed, applying the nabla differential operator to equation (15), in view of the equilibrium equation outside the tube, $\nabla \delta p_{e}=\delta \rho_{e} \mathbf{g}$, we get

$$
\nabla \delta P=\nabla \delta p_{e}=\delta \rho_{e} \mathbf{g}
$$

Equation (16) describes the coupling between internal and external forces since the force $\delta \mathbf{F}_{\perp}$ (see equation (14) ) includes the term $\nabla_{\perp} \delta P$ and, hence, the external gravity force $\delta \rho_{e} \mathbf{g}_{\perp}$.

Musielak and Ulmschneider (2001) in order to prove the consistency of the phenomenological equation (14) and the magnetohydrodynamic principles instead of equation (16) used the following boundary conditions

$$
\nabla \delta P=\nabla \delta p_{e}=\delta \rho_{e} \mathbf{g}-\rho_{e} \frac{\partial \mathbf{v}_{\perp e}}{\partial t} .
$$

Equation (17) differs from equation (16) by the inertia term $\rho_{e} \partial \mathbf{v}_{\perp e} / \partial t$. As a result, Musielak and Ulmschneide (2001) are forced to suppose that the velocities normal to the interface within $\left(\mathbf{v}_{\perp}\right)$ and outside $\left(\mathbf{v}_{\perp e}\right)$ the tube have different signs, i.e. $\mathbf{v}_{\perp}=-\mathbf{v}_{\perp e}$ that doesn't make sense (in particular, it means that authors used different systems of coordinates inside and outside the tube).

In order to investigate the linear kink oscillations of the vertical magnetic flux tubes the elastic thread model was elaborated by Spruit (1981). This model based on equation (14) and the thin magnetic flux tube approximation described in Section (2) gives the following wave equation (Spruit, 1981)

$$
\frac{\partial^{2} s_{\perp}}{\partial z^{2}}-\frac{1}{2 H} \frac{\partial s_{\perp}}{\partial z}+\frac{\omega^{2}}{c_{k}^{2}} s_{\perp}=0,
$$

where $s_{\perp}$ is the displacement perpendicular to the tube axis $z$. The solution of this differential equation is

$$
s_{\perp} \propto e^{1 /(4 H) z \pm i \sqrt{\omega^{2}-\omega_{c}^{2}} / c_{k} z}
$$

It describes the propagation of kink waves with the cutoff frequency $\omega_{c}=c_{k} / 2 H$. 


\section{Power series expansion and thin magnetic flux tube approximation}

Let us consider briefly main features of the approach, which is based on the method of the power series expansion with respect to $r$ (see also Roberts and Webb, 1978; Ferriz-Mas and Schüssler, 1989).

According to this method, the perturbed quantities $f(r, z, t)$ inside a thin magnetic flux tube, using standard notation, can be expanded in a Taylor series as

$$
\begin{gathered}
f(r, z, t)=\quad \\
f(0, z, t)+r \frac{\partial f}{\partial r}(0, z, t)+r^{2} \frac{\partial f}{\partial r}(0, z, t)+\ldots= \\
f_{0}(z, t)+f_{1}(r, z, t)+f_{2}(r, z, t)+\ldots,
\end{gathered}
$$

where $\left|f_{n+1}(z, t)\right| /\left|f_{n}(z, t)\right|=\mu \ll 1$ and $f(r, z, t)$ is a slowly varying function of $z$ and $\varphi$, i.e.

$$
\left|\frac{\partial f}{\partial r}\right| \sim \frac{1}{\mu}\left|\frac{\partial f}{\partial z}\right| \sim \frac{1}{\mu r}\left|\frac{\partial f}{\partial \varphi}\right| .
$$

Note that Lopin and Nagorny (2013) used a Laurent series outside the tube since perturbed quantities outside a magnetic flux tube decrease with $r$ in this region (e.g., Tsap and Kopylova, 2001).

Perturbed quantities inside a thin magnetic flux tube can be represented as

$$
\begin{gathered}
s_{r}(r, \varphi, z)=s_{r 0}(\varphi, z)+s_{r 2}(r, \varphi, z)+\ldots, \\
s_{\varphi}(r, \varphi, z)=s_{\varphi 0}(\varphi, z)+s_{\varphi 2}(r, \varphi, z)+\ldots, \\
s_{z}(r, \varphi, z)=s_{z 1}(r, \varphi, z)+s_{z 3}(r, \varphi, z)+\ldots, \\
b_{r}=b_{r 0}(\varphi, z)+b_{r 2}(r, \varphi, z)+\ldots, \quad b_{\varphi}=b_{\varphi 0}(\varphi, z)+b_{\varphi 2}(r, \varphi, z)+\ldots, \\
b_{z}=b_{z 1}(r, \varphi, z)+b_{z 3}(r, \varphi, z)+\ldots, \\
\delta P=\delta P_{1}(r, \varphi, z)+\delta P_{3}(r, \varphi, z)+\ldots,
\end{gathered}
$$

where the perturbed total pressure $\delta P=\delta p+(\mathbf{b B}) / 4 \pi$. In turn, perturbed quantities outside a magnetic flux tube are described by Hankel or Macdonald functions (e.g. Tsap and Kopylova, 2001). This suggests that the equation of motion outside the tube has the form (Lopin and Nagorny, 2013; Lopin et al., 2014)

$$
\rho_{e} \frac{\partial^{2} s_{r 0}}{\partial t^{2}}=\frac{\delta p_{e 0}}{r}
$$

Thus, using Equations (11), (17), (11), (13), (21)-(27), and a system of linear equations of ideal MHD (e.g. Lopin and Nagorny, 2013), we can find the wave equation of kink modes (Lopin and Nagorny, 2013; Lopin et al., 2014)

$$
\frac{\partial^{2} s_{r 0}}{\partial z^{2}}-\frac{1}{2 H} \frac{\partial s_{r 0}}{\partial z}+\left(\frac{\omega^{2}}{c_{k}^{2}}+\frac{1}{16 H^{2}}\right) s_{r 0}=0,
$$

where the phase velocity $c_{k}=B_{z} / \sqrt{4 \pi\left(\rho+\rho_{e}\right)}$. It is easy to show that the solution of equation (28) can be represented as (Lopin and Nagorny, 2013; Lopin et al., 2014)

$$
s_{r 0} \propto e^{1 /(4 H) z \pm i \omega / c_{k} z} .
$$

Expression (29) as distinguished from (19) corresponds to the cutoff free propagation of kink modes. 


\section{Lopin et al. (2014) vs Spruit (1981)}

Using equation (14), Spruit (1981) has shown that equation of motion perpendicular to the tube axis in curvilinear coordinates has the form

$$
\left(\rho+\rho_{e}\right) \frac{\partial^{2} \mathbf{s}_{\perp}}{\partial t^{2}}=\frac{B^{2}+B_{e}^{2}}{4 \pi} \mathbf{c}+\left(\rho-\rho_{e}\right)\left(\mathbf{e}_{l} \times \mathbf{g}\right) \times \mathbf{e}_{l},
$$

where $\mathbf{e}_{l}$ is the local unit vector oriented along the magnetic-field lines and $\mathbf{c}=\left(\mathbf{e}_{l} \cdot \nabla\right) \mathbf{e}_{l}$ is the curvature vector.

Lopin et al. (2014) in order to reveal disadvantages of Spruit's approach reduces equation (30) to equation (28) obtained by Lopin and Nagorny (2013) in terms of the linear induction equation in Cartesian coordinates

$$
b_{x 0}=\left(B_{z} \frac{\partial s_{x 0}}{\partial z}+\frac{\partial B_{z}}{\partial z} s_{x 0}\right)+\frac{B_{x}}{x} s_{x 0}
$$

where $b_{r 0}=b_{x 0} \cos \varphi$ and $s_{r 0}=s_{x 0} \cos \varphi$ (Lopin et al., 2014). Note that we replace the subscript $x$ by $x_{0}$ as distinguished from the corresponding equation obtained by Lopin et al. (2014) in order to stress that we consider zeroth-order quantities.

In view of equation (7), we have (see also Lopin et al., 2014)

$$
B_{x}(x, z)=-\frac{x}{2} \frac{\partial B_{z}}{\partial z}
$$

and after substitution of equation (32) to the expression (31) the inclination of the perturbed tube axis is

$$
l_{x}^{\prime}=\frac{b_{x 0}}{B_{z}}=\frac{\partial s_{x 0}}{\partial z}+\frac{s_{x 0}}{2 B_{z}} \frac{\partial B_{z}}{\partial z} .
$$

Equation (33) does not coincide with the corresponding expression obtained by Spruit (1981) which can be written as

$$
l_{x}^{\prime}=\frac{b_{x 0}}{B_{z}}=\frac{\partial s_{x 0}}{\partial z} .
$$

Lopin et al. (2014) have shown that equation (30) is reduced to wave equation (28) after substitution equation (33) to equation (30). Whence, comparing equations (33) and (34), they concluded that the main reason of the discrepancy is associated with the radial component of the tube magnetic field $B_{r}$ which was not taken into account by Spruit (1981). We do not agree with this inference. Really, it suggests that the approach proposed by Lopin and Nagorny (2013) can be considered as a generalization of Spruit's results (Spruit, 1981) and at

$$
\left|\frac{1}{s_{x 0}} \frac{\partial s_{x 0}}{\partial z}\right| \ll\left|\frac{1}{2 B_{z}} \frac{\partial B_{z}}{\partial z}\right|=\frac{1}{4 H},
$$

Equation (28) obtained by Lopin and Nagorny (2013) (see also Lopin et al., 2014) should be reduced to Spruit's equation (18). However, if we take into account that $s_{r 0}=s_{x 0} \cos \varphi$ and inequality (35), the wave equation (28) obtained by Lopin and Nagorny (2013) takes the form

$$
\frac{\partial^{2} s_{x 0}}{\partial z^{2}}+\left(\frac{\omega^{2}}{c_{k}^{2}}+\frac{1}{16 H^{2}}\right) s_{x 0}=0,
$$


where we adopt the displacement $s_{x 0}=s_{\perp}$ since $s_{y 0} \equiv 0$ (Lopin et al., 2014). By comparing equations (18) and (36), we can conclude that they are not coincided. Thus, the discrepancy between results obtained by Spruit (1981) and Lopin et al. (2014) can not be caused by the radial component of the equilibrium magnetic field $B_{r}(r, z)$ and the main reason is related to equation of motion (30), which seems to be not quite reasonable (see Section 3).

\section{Discussion and conclusions}

In the present work we check the statement of Lopin et al. (2014) that Sprit's approach based on the phenomenological equation of motion can be used for description of kink waves of thin magnetic flux tubes if we take into account the radial component of the tube in the induction equation. As it follows from the our results this statement is not quite correct since the main problem is related to the phenomenological equation of motion which can not be used for the description of the external force outside the tube. This conclusion also suggests that the the phenomenological equation of motion can not be used to describe the dynamics of thin flux tubes.

Following Lopin and Nagorny (2013) we have confirmed that the low frequency kink waves of thin magnetic flux tubes are cutoff free modes. In particularly, this suggests that these modes generated in the photosphere by convective motions can be responsible for heating and accelerating the fast wind in spite of quite strong reflection in the transition region (Tsap, 2006; Soler et al., 2017). Also they can be very useful for plasma diagnostics and can support the decayless transverse oscillations of coronal loops (Nisticó et al., 2013). However, the thin flux tube approximation has serious restrictions therefore the investigation of the kink mode propagation in the tubes with more complex magnetic configuration is required.

\section{References}

- Cheng J. Equations for the motion of an isolated thin magnetic flux tube // Astron. Astrophys. V. 264. N 1. P. 243-248. 1992.

- Choudhuri A.R. A correction to Spruit's equation for the dynamics of thin flux tubes // Astron. Astrophys. V. 239. N 1-2. P. 335-339. 1990.

- De Pontieu B., Martens P.C.H., Hudson H.S. Chromospheric Damping of Alfvén Waves // Astrophys. J. V. 558. N 2. P. 859-871. 2001.

- Fan Y. Magnetic Fields in the Solar Convection Zone // Liv. Rev. Solar Phys. V. 6. N 1. id.4. 96 P. 2009.

- Fan Y., Fisher G.H., McClymont A.N. Dynamics of emerging active region flux loops // Astrophys. J. V. 436. N 2. P. 907-928. 1994.

- Ferriz-Mas A., Schüssler M.A.V. // Dynamics of magnetic flux concentrations - The secondorder thin flux tube approximation Astron. Astrophys. V. 210. N 1-2. P. 425-432. 1989.

- Fujimura D., Tsuneta S. Properties of Magnetohydrodynamic Waves in the Solar Photosphere Obtained with Hinode // Astrophys. J. V. 702. N 2. P. 1443-1457. 2009.

- Gelfreikh G.B., Tsap Yu.T., Kopylova Yu.G. et al. Variations of Microwave Emission from Solar Active Regions // Astron. Letters V. 30. 489-495. 2004.

- Hollweg J.V. Alfvén waves in the solar atmosphere. II - Open and closed magnetic flux tubes // Solar Phys. V. 70. P. 25-66. 1981.

- Hollweg J. V. Resonances of coronal loops // Astrophys. J. V. 277. P. 392-403. 1984. 
- Howard R., Stenflo J.O. On the Filamentary Nature of Solar Magnetic Fields // Solar Phys. V. 22. N 2. P. 402-417. 1972.

- Jess D.B., Van Doorsselaere T., Verth G. et al. An Inside Look at Sunspot Oscillations with Higher Azimuthal Wavenumbers // Astrophys. J. V. 842. N 1. id. 59. 9 p. 2017.

- Ji H., Cao W., Goode P.R. Observation of Ultrafine Channels of Solar Corona Heating // Astrophys. J. Letters. V. 750. N 1. id. L25. 5 p. 2012

- Longcope D.W., Klapper I. Dynamics of a Thin Twisted Flux Tube // Astrophys. J. V. 488. N 1. P. 443-453. 1997.

- Leake J.E., Arber T.D., Khodachenko M.L. Collisional dissipation of Alfvén waves in a partially ionised solar chromosphere // Astron. Astrophys. V. 442. N 3. P. 1091-1098. 2005.

- Lopin I., Nagorny I. Conditions for Transverse Waves Propagation along Thin Magnetic Flux Tubes on the Sun // Astrophys. J. V. 774. N 2. id. 121. 5 p. 2013.

- Lopin I.P., Nagorny I.G., Nippolainen E. Kink Wave Propagation in Thin Isothermal Magnetic Flux Tubes // Solar Phys. V. 289. N 8. P. 3033-3041. 2014.

- Lopin I., Nagorny I. Kink Waves in Thin Stratified Magnetically Twisted Flux Tubes // Astrophys. J. V. 840. N 1. id. 26.7 p. 2017.

- Moreno-Insertis F., Ferriz-Mas A., Schlüssler M. Enhanced inertia of thin magnetic flux tubes // Astron. Astrophys. V. 312. P. 317-326. 1996.

- Morton R.J., Tomczyk S., Pinto R. Investigating Alfvénic wave propagation in coronal openfield regions // Nat. Comm. V. 6. id. 7813. 2015.

- Musielak Z.E., Ulmschneider P. Excitation of transverse magnetic tube waves in stellar convection zones. I. Analytical approach // Astron. Astrophys. V. 370. P. 541-554. 2001.

- Nisticó G., Nakariakov V.M., Verwichte E. Decaying and decayless transverse oscillations of a coronal loop // Astron. Astrophys. V. 552. id. A57. 6 p. 2013.

- Osin A., Volin S., Ulmschneider P. Propagation of nonlinear longitudinal-transverse waves along magnetic flux tubes in the solar atmosphere. III. Modified equation of motion // Astron. Astrophys. V. 351. P. 359-367. 1999.

- Roberts B., Webb A.R. Vertical motions in an intense magnetic flux tube // Solar Phys. V. 56. P. 5-35. 1978.

- Ryutov D.D., Ryutova M.P. Sound oscillations in a plasma with "magnetic filaments" // Sov. Phys. JETP. V. 43. N 3. P. 491-497. 1976

- Routh S., Musielak Z.E., Hammer R. Conditions for Propagation of Torsional Waves in Solar Magnetic Flux Tubes // Solar Phys. V. 246. N 1. P. 133-143. 2007.

- Rüedi I., Solanki S.K., Livingston W., Stenflo J.O. Infrared lines as probes of solar magnetic features. III - Strong and weak magnetic fields in plages // Astron. Astrophys. V. 263. N 1-2. P. 323-338. 1992.

- Sharykin I.N., Kosovichev A.G. Fine Structure of Flare Ribbons and Evolution of Electric Currents // Astrophys. J. Letters. V. 788. N 1. id. L18. 7 p. 2014.

- Soler R., Terradas J., Oliver R., Ballester J.L. Propagation of Torsional Alfvén Waves from the Photosphere to the Corona: Reflection, Transmission, and Heating in Expanding Flux Tubes Astrophys. J. V. 840. id.20. 18 p. 2017.

- Spruit H.C. Motion of magnetic flux tubes in the solar convection zone and chromosphere // Astron. Astrophys. V. 98. P. 155-160. 1981.

- Stenflo J.O. Collapsed, uncollapsed, and hidden magnetic flux on the quiet Sun // Astron. Astrophys. V. 529. A42. 20 p. 2011. 
- Tsap Yu.T., Kopylova Yu.G. Acoustic Damping of Fast Kink Oscillations of Coronal Loops // Astron. Letters. V. 27. N 11. P. 737-744. 2001.

- Tsap Y.T. On the penetration of Alfvén waves from the chromosphere into the corona // Proc. IAU Symp. N 233, Solar Activity and its Magnetic Origin. Eds. Bothmer V. and Hady A.A. Cambridge Univ. Press, Cambrige, p.253-254. 2006.

- Weber M.A., Browning M.K. Modeling the Rise of Fibril Magnetic Fields in Fully Convective Stars // Astrophys. J. V. 827. id.95. 20 p. 2016. 